\title{
Cytokines, estradiol and progesterone in the plasma of women of reproductive age with pelvic inflammatory disease in remission
}

\author{
Aleksandr Trunov*, Olga Obukhova, Olga Gorbenko, Alya Shvayk, Liliya Trunova \\ Center of Clinical and Experimental medical, Siberian Branch of Russian Academy of Medical Sciences, Novosibirsk, Russia \\ *Corresponding Author: Trunov1963@yandex.ru
}

Received 22 April 2013; revised 25 May 2013; accepted 2 June 2013

Copyright (C) 2013 Aleksandr Trunov et al. This is an open access article distributed under the Creative Commons Attribution License, which permits unrestricted use, distribution, and reproduction in any medium, provided the original work is properly cited.

\begin{abstract}
In the course of the study there were examined cytokine levels of pro-inflammatory and anti-inflammatory activity (IL-1 $\beta$, IL-4, IL-6, IL-8, IL-10, IFN- $\gamma$, G-GSF), and also the levels of progesterone and estradiol in the blood serum with a view to study the pathogenic characteristics of a chronic inflammatory process of the pelvic organs in remission. It was shown that the remission of the disease is characterized by the significant increase in the concentrations of pro-inflammatory cytokines IL-6, IL-8, and also increase of inducers activation of the immune response of the immune response (IL-4 and IFN- $\gamma$ ), accompanied by a decrease in the concentration of sex hormones. In women with chronic inflammatory diseases of urogenital sphere in remission tested active immunological process and violation of the synthesis of sex hormones. Revealed changes predispose to the emergence disorders of reproductive health.
\end{abstract}

Keywords: Immune Response; Sex Hormones; Chronic Inflammation; Remission; Cytokines

\section{INTRODUCTION}

One of the important areas of obstetrics and gynecology, which allows to search for new approaches to the task of increasing reproductive health, is a study aimed at exploring the pathogenesis of chronic infectious and inflammatory diseases of the urogenital areas. Exactly this kind of pathological conditions play a significant role in the development disorders of reproductive health of women [1,2].

Currently, research results allow to speak not only about the rise in the spread of diseases of the urogenital areas of inflammatory nature, but also a high level of chronization of these processes [2,3].

There is no doubt that chronic inflammatory diseases of the pelvic organs in women of reproductive age are an important factor leading to complications of pregnancy, disorders of fetal development. Moreover, chronic processes in urogenital sphere can be a significant risk factor for infertility [4-6].

Certainly, many aspects of immune responses in the pathogenesis of acute and exacerbations of chronic and infectious and inflammatory diseases of the pelvic organs in women studied quite well $[4,7,8]$. Also in acute and exacerbations of chronic inflammation of the pelvic organs in women it was studied the nature of the imbalance of cytokines and their effect on the violation of the synthesis of a number of sex hormones, which play a significant role in reproduction [4,8-10].

At the same time, the results of studies that reflect the nature of immune disorders and the activity of the inflammatory process in the period of remission of chronic inflammatory diseases of the pelvic organs in women are represented by single works. So, in our previous studies data was obtained showing the activity of the inflammatory process in women with remission of chronic inflammatory processes of the pelvic organs [6,11]. But perhaps it is the activity of the inflammatory process, as well as its impact on the production of sex hormones in remission of the pathological process, that largely determines the further development of the disease, its severity and occurrence of reproductive disorders.

All of the above determines the theoretical and practical importance of studies on the in-depth study of the immunopathogenesis of chronic inflammatory diseases of the pelvic organs in remission in women of reproductive age.

The purpose of this article was to study the features of cytokine level changing and dynamics of synthesis of progesterone and estradiol in women of childbearing age 
with chronic inflammatory diseases of the pelvic organs in remission of the process.

\section{MATERIALS AND METHODS}

In accordance with the purpose of the study on the basis of municipal health care institutions "Novosibirsk City Perinatal Center" were examined 65 non-pregnant women aged 20 to 35 years. All women gave informed consent to participate in the study, which is consistent with the ethical standards developed in accordance with the Declaration of Helsinki. Study groups were comparable in age.

The main group (I group) consisted of 50 patients with established diagnosis of chronic inflammatory diseases of urogenital sphere in remission. Inclusion criteria for group I was the presence of chronic urogenital areas of inflammatory disorders in remission. Diagnosis is confirmed by the data of history, clinical, laboratory and instrumental methods. Also, the criteria for inclusion in the core group were the lack of subjective complaints, increase in body temperature, as well as reference values in the peripheral blood (white blood cell count, WBC, ESR level).

The control group (II group) consisted of 15 healthy women between the ages of $20-35$. The criterion for inclusion in the control group of patients was the lack of a history of chronic inflammatory diseases of different organs and systems, including the pelvis. In addition, the criteria for inclusion in the control group of women surveyed were the lack in the three months before the examination of any acute inflammatory diseases of bacterial and viral etiology.

Exclusion criteria for both groups studied was the presence of women of any endocrine disorders that can affect the synthesis of sex hormones and exerts influence on the functional state of the immune system, as well as autoimmune and malignant neoplastic processes.

Determination of the concentrations of interleukins IL-1 $\beta$, IL-4, IL-6, IL-8, IL-10, interferon gamma (IFN- $\gamma$ ) and granulocyte growth factor (G-GSF) in serum was carried out using commercial test systems for immunoassay produced by "Cytokine" company (Russia) at the manufacturer's instructions.
Determination of levels of estradiol in the blood serum was carried out on 3 - 5 day menstrual cycle. Determination of progesterone in serum was carried out at $22-24$ days of the menstrual cycle. To determine the levels of sex hormones there were used commercial test systems for immunoassay production DRG Diagnostics (Germany) at the manufacturer's instructions.

ELISA results were recorded on a vertical photometer "Uniplan" at a wavelength of $450 \mathrm{~nm}$.

The resulting digital data were statistically analyzed and are presented in tabular form. Data analysis was performed using the package of applied programs Statgrafics. The significance of differences in the variation series of unrelated samples was evaluated using the Mann-Whitney test, the related pairs samples - using Wilcoxon test. Data in tables are presented as $\mathrm{M} \pm \mathrm{m}$, where $\mathrm{M}-$ mean, $\mathrm{m}$ - error of the mean. Considered significant difference between treatment series with a confidence level of $95 \%(\mathrm{p}<0.05)$.

Correlation analysis was carried out using Spearman rank correlation.

\section{RESULTS AND DISCUSSION}

In assessing the cytokines in the serum of patients I and II revealed the following trends, which are presented in Table 1.

The average concentration of the pro-inflammatory cytokine-IL-1 $\beta$ in the serum of patients of the main group had a tendency to rise in the relative values of the control group, but they are not significantly different. This lack of significant differences in the patient confirms the absence of clinical manifestations of group I of inflammatory activity.

However, it showed a significant $(\mathrm{p}<0.05)$, more than 1.6 times increase in IL- 8 in patients in group I relatively to the values obtained in the control group. This increase in IL-8 in serum of patients with remission of chronic inflammation of the pelvic organs may be explained by a function of IL-8, which is a chemoattractant and an active participant in the development of local inflammatory reactions. These data may indicate that, despite the absence of clinical manifestations of inflammation of the urogenital areas in remission in patients in group I, at the

Table 1. Content of IL-1 $\beta$, IL-8, IL-4, IL-6, IFN- $\gamma$, IL-10, G-GSF in the serum of patients with remission of chronic inflammation of the pelvic organs and healthy women $(\mathrm{M} \pm \mathrm{m})$.

\begin{tabular}{|c|c|c|c|c|c|c|c|}
\hline \multirow{2}{*}{ Groups } & \multicolumn{7}{|l|}{ Index } \\
\hline & IL- $1 \beta \mathrm{pg} / \mathrm{ml}$ & IL-8 pg/ml & IL-4 pg/ml & IL-6 pg/ml & IFN $-\gamma \mathrm{pg} / \mathrm{ml}$ & IL-10 pg/ml & G-GSF pg/ml \\
\hline $\begin{array}{c}\text { Group I-main } \\
\text { (remission of chronic } \\
\text { inflammation) } \\
\mathrm{n}=50\end{array}$ & $56.7 \pm 12.1$ & $59.3 \pm 6.6^{*}$ & $82.3 \pm 9.1^{*}$ & $62.3 \pm 6.2^{*}$ & $60.9 \pm 9.4^{*}$ & $22.4 \pm 5.1$ & $48.2 \pm 9.2^{*}$ \\
\hline $\begin{array}{c}\text { Group II-control } \\
\text { (healthy women) } \\
\mathrm{n}=15\end{array}$ & $47.4 \pm 9.3$ & $36.6 \pm 4.6$ & $38.5 \pm 6.3$ & $28.9 \pm 4.3$ & $32.8 \pm 6.8$ & $16.2 \pm 2.5$ & $86.8 \pm 15.0$ \\
\hline
\end{tabular}

*Significantly different from the standard values of parameters $(\mathrm{p}<0.05)$. 
local level continues to actively develop a destructive inflammatory process.

The average content of the pro-inflammatory cytokine IL-6, involved in the processes of chronization, in the serum of patients of the main group was significantly higher, by 2.2 times, than the values, obtained in the control group $(p<0.05)$. It is also known that IL-6 is capable of at certain stages of development of the process of inflammation, suppress the synthesis of IL- $1 \beta$ and strengthen the processes of antibody in the second phase of the immune response.

It is known that IL-4 acts as an inductor of humoral immune system by activating T-helper- 2 mediated immune response, the development of which is characteristic, including, for bacterial infection processes. In the study of IL-4 content in the serum of women surveyed, it was found it's significantly higher level in the main group, compared with the control group. The content of IL-4 in patients in group I is more than 2-fold greater than those studied in the control group $(p<0.05)$. Tested in the study, high concentrations of IL- 6 and IL- 4 can be seen as a reaction of the immune system in order to stimulation of antibody and activation of the humoral immune response in response to the persistence of pathogens from the source of the pathological process of chronic inflammation, that is, the development of inflammatory reactions, despite the absence of clinical manifestations.

In determining the content of endogenous immunostimulant IFN- $\gamma$, noted that in the serum of patients in group I with chronic inflammation, the level of IFN- $\gamma$ is 1.9 times higher than the values studied in the control group of healthy women, and this increase was significant $(\mathrm{p}<0.05)$. It is known that IFN- $\gamma$ plays an important role in the development of a specific phase of the immune response. Therefore, determined increasing IFN$\gamma$ in conjunction with the absence of clinical signs of chronic inflammation of the pelvic organs may indicate the activation of a specific immune response as a response to antigen stimulation of the inflammatory focus.

In assessing the content of granulocyte colony-stimulating factor (G-GSF), which determines the activity of phagocytes immune system, noted the average value of G-GSF in the serum of patients of the main group was significantly, by 1.8 times lower than the values specified in the control group $(p<0.05)$. These data suggest that the mechanisms of chronization of inflammatory diseases of the urogenital areas of women of reproductive age, significant role plays a phagocytes functional failure of the immune system.

Mean concentrations of IL-10 in the serum of patients of the main group had a tendency to increase the relative value of the index in the control group, but they are not significantly different.

Given that there is evidence of the influence of inflammatory processes in the production of various hormones in the present study was conducted determine estradiol and progesterone-sex hormones, involved in the regulation of fertilization and gestation. The research resulted in the following findings. Mean estradiol on 3 5 day of the menstrual cycle in the blood serum of patients of the main group was $198.1 \pm 14.2 \mathrm{pg} / \mathrm{ml}$, which was significantly lower than the rate obtained in the control group $-261.2 \pm 24.1 \mathrm{pg} / \mathrm{ml}(\mathrm{p}<0.05)$.

Average progesterone levels by $22-24$ days of the menstrual cycle in the blood serum of patients of the main group was $4.7 \pm 0.4 \mathrm{ng} / \mathrm{ml}$, which was significantly lower $(1.3$-fold $)$ than the values obtained in the control group- $6.1 \pm 0.5 \mathrm{ng} / \mathrm{ml}(\mathrm{p}<0.05)$.

These data seem to suggest that the presence of chronic inflammatory process of urogenital sphere in women of fertility age reduces estradiol and progesterone, sex hormones that determine women's reproductive health, and this reduction remains in remission of the inflammatory process.

The study carried out a correlation analysis of the data (Table 2).

Identified correlative relationships suggest a correlation between the activity of the inflammatory process and increase of the synthesis of inducers of an immune response in women of reproductive age with chronic inflammatory diseases of the pelvic organs in remission.

However, the most significant of the identified relationships is inverse correlative relationship between the concentration of IL-8 and progesterone level, which attests, that the presence of inflammatory-destructive process of the pelvic organs in women of fertility age is the reason for the inhibition of progesterone synthesis, which is likely to be one of the mechanisms of reproductive health disorders.

Summarizing the data, we can draw the following conclusion. We surveyed women of reproductive age with chronic inflammatory diseases of the pelvic organs in

Table 2. Correlations of indicators in the group of patients with remission of chronic inflammation of the pelvic organs.

\begin{tabular}{cccc}
\hline Indicator 1 & Indicator 2 & $\begin{array}{c}\text { Correlation } \\
\text { coefficient }(\mathrm{r})\end{array}$ & $\begin{array}{c}\text { Significance of } \\
\text { the difference }(\mathrm{p})\end{array}$ \\
\hline IL-1 $\beta$ & IL-6 & $\mathrm{r}=0.41$ & $\mathrm{p}<0.02$ \\
IL-1 $\beta$ & IL-8 & $\mathrm{r}=0.37$ & $\mathrm{p}<0.05$ \\
IL-4 & IFN- $\gamma$ & $\mathrm{r}=0.41$ & $\mathrm{p}<0.02$ \\
IL-4 & IL-6 & $\mathrm{r}=0.54$ & $\mathrm{p}<0.05$ \\
IL-6 & IFN- $\gamma$ & $\mathrm{r}=0.54$ & $\mathrm{p}<0.05$ \\
IL-8 & progesterone & $\mathrm{r}=-0.45$ & $\mathrm{p}<0.05$ \\
\hline
\end{tabular}


remission tested increasing concentrations of pro-inflammatory cytokines IL-6 and IL-8. Also in the examined patients revealed a significant increase of the concentrations of inducers of humoral and cellular immune response-IL-4 and IFN- $\gamma$, amid falling granulocyte colony-stimulating factor (G-GSF), regulating the functional activity of phagocytes immune system. The data indicate that the remission of the pathological process in the women studied, in the absence of clinical signs, is determined the activity of immunoinflammatory reactions. Given that the permanent stimulation of the immune reactivity can with time deplete the adaptation reserves, and, therefore, lead to the development of immune deficiency and is the basis for the emergence of periodic exacerbations of the pathological process.

Unfortunately, implemented scientific research did not allow us to find similar papers about the imbalance of sex hormones and cytokines in their relationship with remission of chronic inflammation of the pelvic organs in women of reproductive age. This did not allow us to make a comparative analysis of obtained data.

Results of our research shows that the presence of chronic inflammation of the reproductive system can initiate, both the development of endocrine disorders, manifested by reduced levels of estradiol and progesterone, and also create unfavorable by the appearance of pregnancy and cytokine balance, whose role in reproduction is not in doubt. Thus, the presence of source of chronic inflammation of the pelvic organs, even without clinically significant symptoms in the future, without sanitizing activities can initiate the development of reproductive health conditions and be the basis for the emergence of infertility and miscarriage and have a significant negative impact on women's reproductive health.

The study was conducted using the equipment access center "Modern optical systems" Research Center of Clinical and Experimental medical, Siberian Branch of Russian Academy of Medical Sciences.

\section{REFERENCES}

[1] Kondranina T.G., Gorin V.S. and Grigoriev E.V. (2009), Acute-phase proteins and markers of endotoxemia, and their prognostic value in gynecological practice. Russian Journal of Obstetrician and Gynecologist, 9, 26-30.

[2] Smith, M., Hagerty, K.A., Skipper, B. and Bocklage, T. (2010) Chronic endometritis: A combined histopathologic and clinical review of cases from 2002 to 2007. Interna- tional Journal of Gynecological Pathology, 1, 44-50. doi:10.1097/PGP.0b013e3181ae81bb

[3] Frolov, M.V. and Khalim H. (2006) Evaluation of the reproductive function of women with acute and chronic inflammatory diseases of the uterus. Journal of Voronezh, 7, 21-26.

[4] Mardh, P.A. (2004) Tubal factor infertility, with special regard to chlamydial salpingitis. Current Opinion in Infectious Diseases, 1, 49-52. doi:10.1097/00001432-200402000-00010

[5] Gorin, V.S., Saginor, M.E., Maltiyskaya, N.A., Birjukova, L.A. and Maltiysky, M.L. (2008) Principles of diagnosis and treatment of purulent inflammatory diseases of the uterus. Russian Journal of Obstetrician and Gynecologist, 5, 30-38.

[6] Trunova, L.A., Gorbenko, O.M., Shvayk, A.P., Obukhova, O.O., Anisimova, N.I., Chernikh, V.V., Safronov, I.D. and Trunov A.N. (2008) Active immunological process in women of reproductive age with chronic infectious and inflammatory diseases of the uterus in clinical remission. Bulletin of SO RAMS, 1, 80-83.

[7] Strijakov, A.N., Kargamanova, J.A. and Yakubovich, D.V. (2004) Clinical and immunological study of therapy in patients with acute inflammation of the uterus. Questions of Gynecology, Obstetrics and Perinatology, 3, 26-29.

[8] Lee, S.A., Tsai, H.T., Ou, H.C., Han, C.P., Tee, Y.T., Chen, Y.C., Wu, M.T., Chou, M.C., Wang, P.H. and Yang, F. (2008) Plasma interleukin-1beta, -6, -8 and tumor necrosis factor-alpha as highly informative markers of pelvic inflammatory disease. Clinical Chemistry and Laboratory Medicine, 7, 997-1003.

[9] Ng, S.C., Gilman-Sachs, A. and Thaker, P. (2002) Expression of intracellular Th1 and Th2 cytokines in women with recurrent spontaneous abortion, implantation failures after IVF/ET or normal pregnancy. American Journal of Reproductive Immunology, 2, 77-86. doi:10.1034/j.1600-0897.2002.01105.x

[10] Chen, K.S., Wang, P.H., Yang, S.F., Lin, D.B., Lin, Y.J., Kuo, D.Y., Lin, L.Y., Wu, M.T., Lin, C.W., Lee, S, Chou, M.C., Tsai, H.T. and Hsieh, Y.S. (2008) Significant elevation of a Th2 cytokine, interleukin-10, in pelvic inflammatory disease. Clinical Chemistry and Laboratory Medicine, 11, 1609-1616.

[11] Trunov, A.N., Efremov, A.V. and Trunova, L.A. (2002) Principles pathogenetically substantiated diagnosis in therapies of chronic infectious and inflammatory diseases. Allergology and Immunology, 1, 117. 